\title{
Resonance-assisted parametric electron pump
}

\author{
Yadong Wei, ${ }^{1,2}$ Jian Wang, ${ }^{1}$ and Hong Guo ${ }^{3}$ \\ ${ }^{1}$ Department of Physics, The University of Hong Kong, Pokfulam Road, Hong Kong, China \\ ${ }^{2}$ Department of Physics, Shanxi University, Taiyuan, Shanxi 030006, China \\ ${ }^{3}$ Centre for the Physics of Materials and Department of Physics, McGill University, Montreal, PQ, Canada H3A 2T8
}

(Received 15 February 2000; revised manuscript received 22 May 2000)

\begin{abstract}
We report a theoretical analysis of parametric quantum pumping of electric current which is aided by quantum resonance. The electron pump is realized by cyclic deformations of the barrier heights of a doublebarrier quantum well. The pumped current is found to have large values near a resonant level, it has a rather sensitive dependence on such control parameters as the deformation strength, phase difference, and the well width, and it has a power-law temperature dependence.
\end{abstract}

A parametric electron pump drives an electric current at zero bias by cyclic deformations of two or more system parameters. $^{1,2}$ Classical pumps have been reported to operate in the Coulomb blockade (CB) regime, ${ }^{3,4}$ where electrons are forced through the device by cycling the coupling of the device to the reservoirs. More recently, on open quantumdot-based parametric electron pump has been fabricated ${ }^{5}$ where two gates with oscillating voltages control the deformation of the shape of the dot. The pumped dc voltage $V_{\text {dot }}$ is measured to vary with the phase difference $\phi$ between the two gate voltages, and is antisymmetric about $\phi=\pi$. At low pumping amplitude, the experimental data gave $V_{\text {dot }} \sim \sin \phi$. The amplitude of the pumped signal is found to increase nonlinearly with the driving force, and it decays with temperature $T$ as a power law $\sim 1 / T^{0.9}$. Motivated by the very interesting findings of the experiment, ${ }^{5}$ in this paper we report a theoretical analysis of the adiabatic quantum electron pumping phenomenon in a double-barrier quantum-well device.

The general physics of a quantum electron pump has been the subject of several theoretical analyses, ${ }^{6,2,7}$ and the device has been proposed to become an electric current standard. ${ }^{8}$ However, to date all theoretical investigations of parametric pumping have focused on open and transparent device structures. For electron pumps operating in the $\mathrm{CB}$ regime, the energy level spacing $\Delta E=E_{i+1}-E_{i}$ of the device is in general much smaller than the charging energy, where $E_{i}$ is the $i$ th single electron level. Therefore, in the $\mathrm{CB}$ regime $\Delta E$ is irrelevant to the pumping operation. On the other hand, this paper examines the phenomenon of resonance-assisted electron pumping for which $\Delta E$ plays the most important role. We will be interested in the well known resonance tunneling regime ${ }^{9}$ for which charging energy is of no concern although the device is not transparent. Our results indicate that electron pumping is drastically modified by the resonance states such that the pumped current obtains a very large value at a resonance point. As the Fermi energy is varied (which can be controlled by gate voltage), the pumped current can reverse its direction as a result of competition between two pumping parameters. Our result shows that the pumped current is antisymmetric about $\phi=\pi$, consistent with the experiment of Ref. 5. In the strong pumping regime, the calculated pumped current shows a nonsinusoidal dependence of the phase difference, similar to the experimental findings. ${ }^{5}$

To analyze parametric quantum pumping, we make use of the scattering theory developed by Brouwer ${ }^{7}$ where an electron-electron interaction such as the Coulomb blockade effect is neglected. In order for a parametric electron pump to function, we need simultaneous variation of two system parameters $X_{1}(t)=X_{1} \sin (\omega t)$ and $X_{2}(t)=X_{2} \sin (\omega t+\phi)$. If the time variation of these parameters is slow, i.e., $X(t)$ $=X_{0}+\delta X \sin (\omega t)$, one can prove ${ }^{7}$ that the charge passing through contact $\alpha$ due to the infinitesimal change of the system parameter $(\delta X \rightarrow 0)$ is

$$
d Q_{\alpha}(t)=q \frac{d N_{\alpha}}{d X_{1}} \delta X_{1}(t)+q \frac{d N_{\alpha}}{d X_{2}} \delta X_{2}(t) .
$$

Furthermore, the current flowing through contact $\alpha$ due to the variation of parameters $X_{1}$ and $X_{2}$, in one period of time, is given $\mathrm{by}^{7}$

$$
I_{\alpha}=\frac{q \omega}{2 \pi} \int_{0}^{\tau} d t\left[\frac{d N_{\alpha}}{d X_{1}} \frac{d X_{1}}{d t}+\frac{d N_{\alpha}}{d X_{2}} \frac{d X_{2}}{d t}\right],
$$

where $\tau=2 \pi / \omega$ is the period of cyclic variation. The quantity $d N_{\alpha} / d X$ is the emissivity ${ }^{10}$ which, in scattering matrix theory, is determined by the following expression:

$$
\frac{d N_{\alpha}}{d X}=\int \frac{d E}{2 \pi}\left(-\partial_{E} f\right) \sum_{\beta} \operatorname{Im} \frac{\partial S_{\alpha \beta}}{\partial X} S_{\alpha \beta}^{*},
$$

where $S_{\alpha \beta}$ is the scattering matrix and $f$ is the Fermi distribution function. The emissivity $d N_{\alpha} / d X$ is the partial density of states (PDOS) in the configurational space for electrons emitted through lead $\alpha$. For instance, if $X$ is energy, then $d N_{\alpha} / d E$ is the familiar PDOS measuring the electron dwell time.

The quantum-well structure ${ }^{11}$ we examine is modeled by potential $U(x)=V_{1} \delta(x+a)+V_{2} \delta(x-a)$, where $V_{1}$ and $V_{2}$ are constants and $2 a$ is the well width. For this system, the Green's function $G\left(x, x^{\prime}\right)$ can be calculated exactly. ${ }^{12}$ This is done by applying Dyson's equation regarding the fact that any one of the $\delta$ barriers is just a perturbation of the remaining system. This way $G\left(x, x^{\prime}\right)$ is obtained by applying Dyson's equation twice, starting from the Green's function 


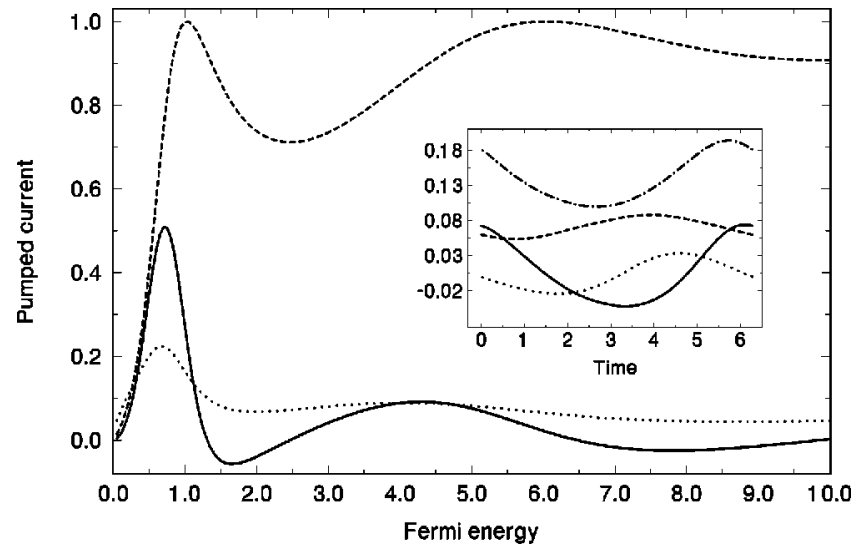

FIG. 1. The pumped current (solid line), emissivity (dotted line), and transmission coefficient (dashed line) versus Fermi energy with $V_{0}=1.0$. For illustrating purpose, we have multiplied $I_{p}$ by a factor of 10. Other parameters are $a=1, \phi=\pi / 2$, and $V_{p}=0.4$. Inset: $q_{1}, q_{2}, d N / d X_{1}$, and $d N / d X_{2}$ versus time in a cycle for $E_{F}=1.6$.

of the one-dimensional free space. With $G\left(x, x^{\prime}\right)$ we can calculate the scattering matrix exactly from the Fisher-Lee relation $^{13} s_{\alpha \beta}=-\delta_{\alpha \beta}+i \hbar v G\left(x_{\alpha}, x_{\beta}\right)$, with $v$ the electron velocity in the lead. Finally, applying Eqs. (1)-(3), the parametric pumping properties can be calculated exactly for the model double-barrier quantum well.

The electron pump we consider is operated by changing barrier heights adiabatically and periodically: $X_{1} \equiv V_{1}=V_{10}$ $+V_{11} \sin (\omega t)$ and $X_{2} \equiv V_{2}=V_{20}+V_{21} \sin (\omega t+\phi)$. This can be achieved by microfabricating metallic gates at the barrier region and applying a time-dependent gate potential. Since the pumped current is proportional to $\omega$ [see Eq. (2)], we will set $\omega=1$ for convenience. We will make a further simplification, without losing generality, by assuming $V_{11}=V_{21}=V_{p}$ and $V_{10}=V_{20}=V_{0} \cdot{ }^{14}$ Finally, the unit is set by $\hbar=2 m=k_{B}$ $=1$, where $k_{B}$ is the Boltzmann constant. For the system of GaAs with $a=100 \AA$, the energy unit is $E=5.6 \mathrm{meV}$, which corresponds to the temperature $T=65 \mathrm{~K}$.

In Fig. 1, we plot the pumped current $I_{p}$ at zero temperature versus the Fermi energy $E_{F}$ (solid line) at $\phi=\pi / 2$. For comparison, we also plot transmission coefficient (dashed line) and emissivity $d N_{\alpha} / d X \equiv \Sigma_{i} d N_{\alpha} / d X_{i}$ (dotted line). The two peaks in the transmission coefficient indicate quantum resonance at those energies mediated by the resonance states. The resonance states have long lifetimes as indicated by the peaks of emissivity. Clearly, the pumped current $I_{p}$ also shows a resonance behavior, sharply peaked at the resonance states. $I_{p}$ is largely suppressed away from resonance, hence in this device it is aided by quantum resonance states. Note that $I_{p}$ comes from a totally different physical mechanism as compared with the conventional tunneling current through the quantum well, ${ }^{9}$ and it is calculated from totally different expressions [see Eq. (2)]. Nevertheless, since $I_{p}$ is quantum coherent, it is subjected to quantum interference, which establishes the resonance behavior. We have confirmed that this is a generic result for the tunneling regime by investigating barriers with different height $V_{0}$, and, in particular, for larger $V_{0}$ the resonances of $I_{p}$ become significantly sharper.

For a conventional tunneling current, its value is either positive or negative once the bias voltage is fixed. However,

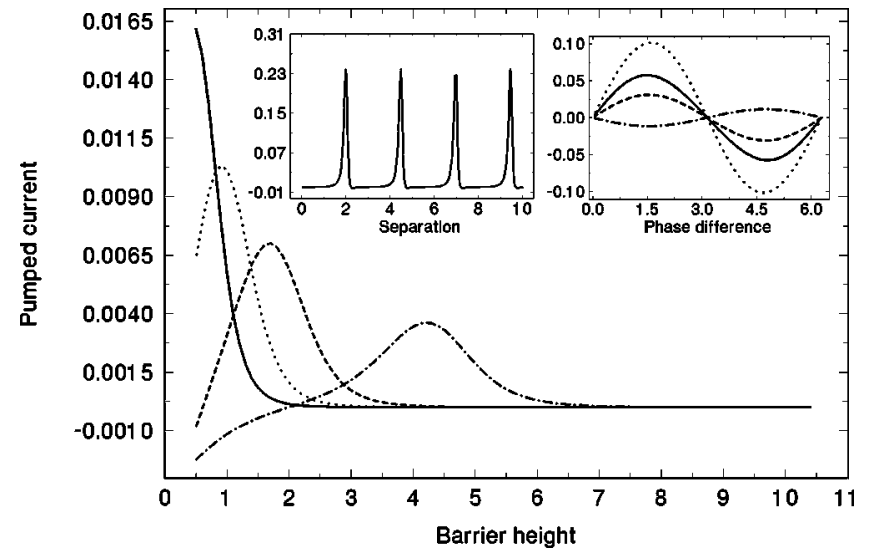

FIG. 2. The pumped current versus barrier height $V_{0}$ for different Fermi energies. Solid line: $E_{F}=0.45$; dotted line: $E_{F}=0.75$; dashed line: $E_{F}=1.1$; dash-dotted line: $E_{F}=1.65$. Other parameters are $a=1, \phi=\pi / 2$, and $V_{p}=0.4$. First inset (the left): the pumped current versus separation $2 a$ with $E_{F}=1.6$ and $V_{0}=4$. Second inset: The pumped current versus phase difference $\phi$ for the four different Fermi energies at $V_{0}=1.0$. We have multiplied $I_{p}$ in the insets by a factor of 10 .

$I_{p}$ can reverse its direction as a function of energy. This is seen in Fig. 1, where $I_{p}$ takes a negative value near $E=1.6$ and 8 , and is a direct consequence of the pumping mechanism. To understand, let us examine the charge pumped through at $E=1.6$. The inset of Fig. 1 shows $q_{1}(t)$ (solid line), $q_{2}(t)$ (dotted line), emissivities $d N / d X_{1}$ (dash-dotted line), and $d N / d X_{2}$ (dashed line) as a function of time. Here $q_{i}=d N / d X_{i} d X_{i} / d t(i=1,2)$ is the charge emitted to the left lead due to the variation of $X_{i}$. Since we have fixed $\phi$ $=\pi / 2$ and $\omega=1$, we obtain $q_{1}=V_{p} d N / d X_{1} \cos (t)$ and $q_{2}=$ $-V_{p} d N / d X_{2} \sin (t)$. Indeed, the numerically calculated $q_{1}$ and $q_{2}$ look very close to $\cos$ and sin functions. The result shows $d N / d X_{1}<d N / d X_{2}$, hence $I_{p} \equiv \int_{0}^{\tau}\left(q_{1}+q_{2}\right) d t / \tau$ is negative at this energy $E=1.6$. Exactly the same way, one can confirm that a positive $I_{p}$ is obtained when $d N / d X_{1}$ $>d N / d X_{2}$.

The pumped current through the double-barrier quantum well is quite sensitive to several system parameters because it is dominated by quantum resonance. Figure 2 shows $I_{p}$ versus the static barrier height $V_{0}$ for several energies. As $V_{0}$ increases, the resonant level inside the quantum well also increases (see the inset of Fig. 4, where we plot the resonant energy as a function of static barrier height). When the resonant level for certain values of $V_{0}$ is in line with the electron Fermi energy, a peak in $I_{p}$ emerges as Fig. 2 depicts. The first inset of Fig. 2 plots $I_{p}$ versus the quantum-well width $2 a$, showing a periodic function of $2 a$. This periodic dependence on well width is actually expected from the form of the scattering matrix. ${ }^{12}$ Essentially, the separation $2 a$ controls the resonant level position which gives rise to the resonances shown in the inset. The second inset of Fig. 2 shows $I_{p}$ as a function of phase difference $\phi$ for different Fermi energies. Our result suggests $I_{p}$ being antisymmetric about $\phi=\pi$ and $\left|I_{p}\right|$ is maximum at $\phi=\pi / 2 . I_{p}$ is reversed if the phase difference between $X_{1}$ and $X_{2}$ is reversed. Note that our result of $I_{p}$ versus $\phi$ is in agreement with the experimental data of Ref. 5.

Experimentally, two important factors are temperature 


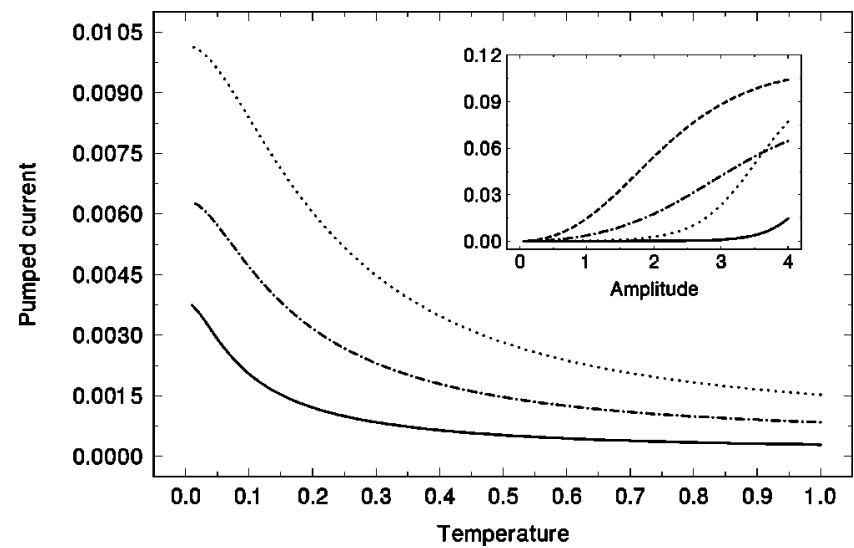

FIG. 3. $I_{p}$ versus temperature $T$ for $V_{0}=4$ and $E_{F}=1.6$ (solid line), $V_{0}=2$ and $E_{F}=1.15$ (dot-dashed line), and $V_{0}=1$ and $E_{F}$ $=0.7$ (dotted line). Other parameters are $a=1, \phi=\pi / 2$, and $V_{p}$ $=0.4$. Inset: $I_{p}$ versus the pumping amplitude $V_{p}$ for different Fermi energies with $V_{0}=4.0$. Solid line: $E_{F}=0.5$; dotted line: $E_{F}$ $=1.0$; dashed line: $E_{F}=1.5$; dash-dotted line: $E_{F}=1.8$.

and pumping amplitude. Temperature smears quantum interference and reduces resonance peaks. Figure 3 shows how $I_{p}$ decreases. The inset of Fig. 3 plots $I_{p}$ versus the pumping amplitude $V_{p}$ for several different Fermi energies. As $V_{p}$ increases, $I_{p}$ increases in a nonlinear fashion. This is also related to the resonance nature. Consider the resonance level $E_{r}$ (for Fig. 3, $E_{r}=1.65$ ). Since the barrier height changes with time, we expect $E_{r}(t)$ to oscillate around $E_{r}$ with an amplitude proportional to $V_{p}$. For $E_{F}=0.5$ (solid line in the inset of Fig. 3), which is far from $E_{r}(t)$ most of the time, it is very difficult for electrons to flow out of the system, hence $I_{p} \approx 0$ unless $V_{p}$ is very large. When $E_{F}$ increases to 1.0, which is closer to $E_{r}, I_{p}$ (dotted line) starts to increase sharply around $V_{p}=2.0$, where the resonant level is about 1.3 according to the inset of Fig. 4. For $E_{F}=1.5$, which is near $E_{r}, I_{p}$ (dashed line) rises very quickly. However, when $E_{F}=1.8$ (dot-dashed line), which is off-resonant again, the pumping is not as efficient as that for $E_{F}=1.5$. When $E_{F}$ $>2.0$, the direction of the pumped current reverses. Finally, we consider the strong pumping case with much larger barrier height. Figure 4 plots the pumped current and the trans-

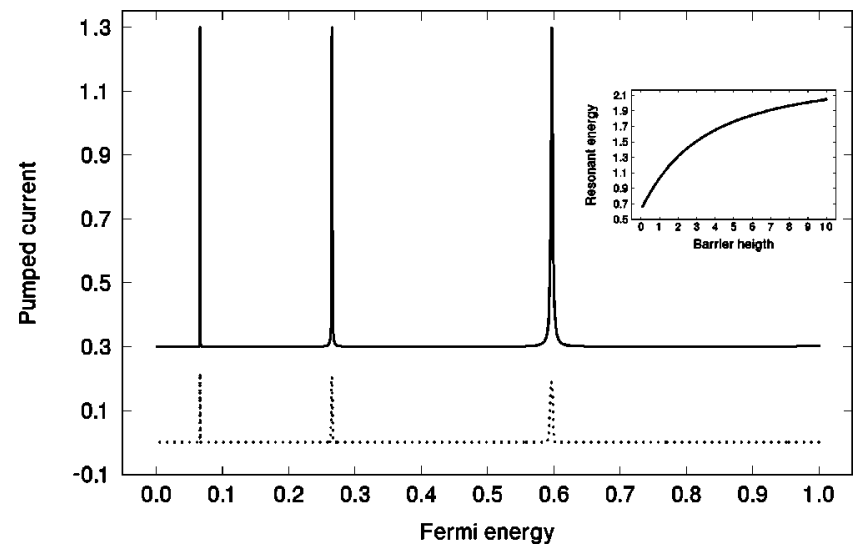

FIG. 4. $I_{p}$ versus $E_{F}$ for $V_{0}=10, V_{p}=2.0, \phi=\pi / 2$, and $a=6$ (solid line) and the corresponding transmission coefficient (dotted line). The transmission coefficient is offset by 0.3 . Inset: the resonant levels $E_{r}$ versus static barrier height $V_{0}$ with $a=1$.
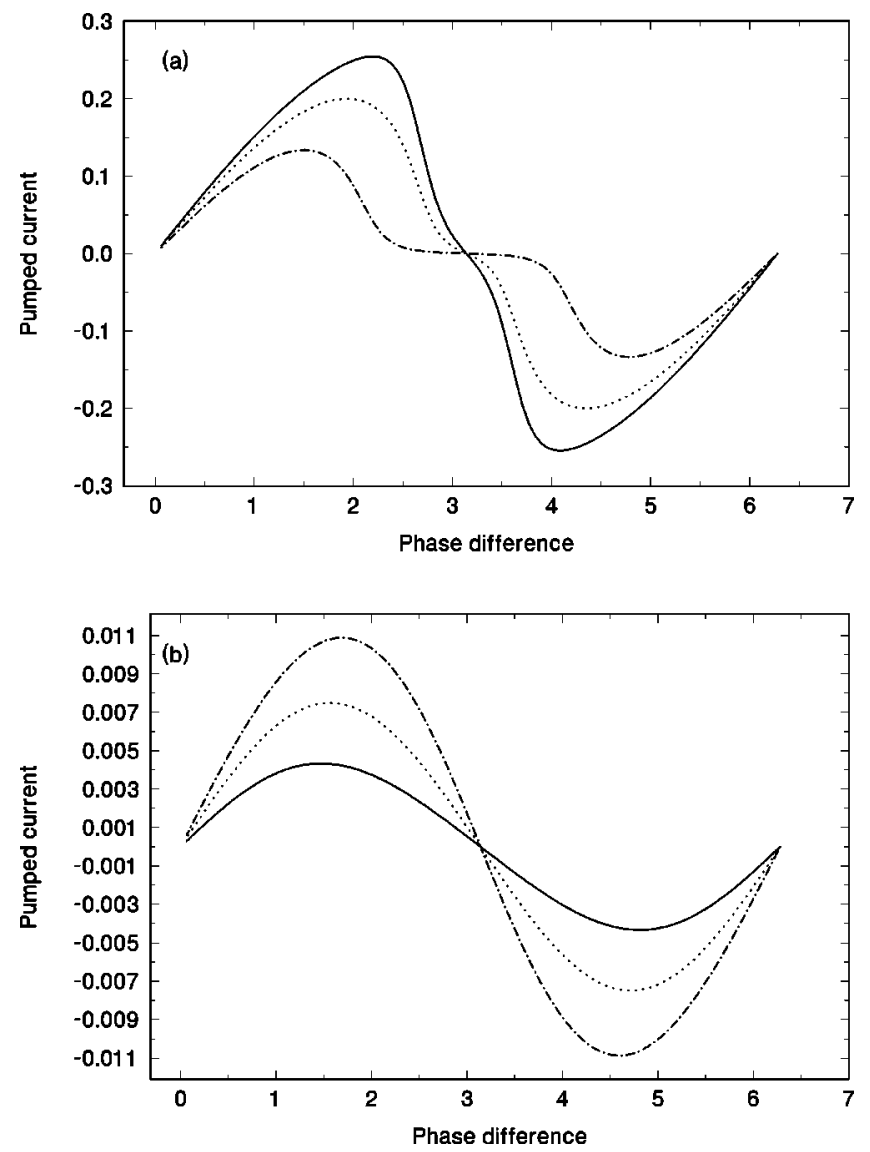

FIG. 5. $I_{p}$ versus $\phi$ at different Fermi energies for $V_{0}=10, a$ =6. (a) $V_{p}=2.0, E_{F}=0.06626$ (solid line), $E_{F}=0.06628$ (dotted line), and $E_{F}=0.06631$ (dash-dotted line). (b) $V_{p}=0.2, E_{F}$ $=0.0662$ (solid line), $E_{F}=0.0664$ (dotted line), and $E_{F}=0.0665$ (dash-dotted line).

mission coefficient for the barrier height $V_{0}=10$ and pumping amplitude $V_{p}=2.0$. Figure 5 presents the pumped current as a function of phase difference. For the strong pumping case with $V_{p}=2.0$ [Fig. 5(a)], the relation between pumped current and the phase difference is no longer sinusoidal while the sinusoidal behavior is maintained at the small pumping amplitude with $V_{p}=0.2$. We note that the experimental data of Ref. 5 also showed a nonlinear driving force dependence by the pumped signal and the nonsinusoidal behavior at strong pumping, although the physical origin is perhaps different from our case studied here. So far, our calculation assumes that the phase coherence of the electron wave is maintained. We have not considered a dephasing mechanism such as inelastic scattering of the phonons. Phenomenologically, the decoherent effects can be modeled by an additional fictitious voltage probe ${ }^{15}$ and treat transmission as a sum of the coherent and incoherent parts. Alternatively, the inelastic-scattering effect can be modeled by including a complex potential in the scattering region. ${ }^{16}$ In general, the interference pattern is smeared out with the increase of the inelastic-scattering rate and hence pumped current as it is due to the large DOS. In the experiment of Ref. 5, the pumped voltage is of order $10^{-7} \mathrm{~V}$ and the conductance is around $2 e^{2} / h \sim(13 \mathrm{k} \Omega)^{-1}$. Hence the pumped current is about $10^{-11}$ A. Note that the scattering theory for parametric pumping is only valid for the first order in frequency 
(compare with the high-frequency limit of $10 \mathrm{THz}$ ). If we take pumping frequency $\omega=100 \mathrm{MHz}$, then our prediction to the pumped current will be around $10^{-11} \mathrm{~A}$.

In summary, for the double-barrier quantum well the pumped current is predicted to behave in a resonant fashion, similar to that of the tunneling current for the device. Our results suggest that a double-barrier quantum well in the resonance tunneling regime is an ideal system for investigating the physics of parametric pumping. This is because the pumped current is predicted to have a distinct dependence on such device parameters as static barrier height, quantum-well width, relative phase, pumping amplitudes, and temperature. All of these can be very well controlled experimentally using present technology.

\section{ACKNOWLEDGMENTS}

We gratefully acknowledge support by a CRCG grant from the University of Hong Kong. H.G. is supported by NSERC of Canada and FCAR of Québec. We thank the Computer Center of the University of Hong Kong for computational facilities.
${ }^{1}$ D.J. Thouless, Phys. Rev. B 27, 6083 (1983).

${ }^{2}$ F. Zhou, B. Spivak, and B.L. Altshuler, Phys. Rev. Lett. 82, 608 (1999).

${ }^{3}$ L.P. Kouwenhoven, et al., Phys. Rev. Lett. 67, 1626 (1991).

${ }^{4}$ H. Pothier, P. Lafarge, C. Urbina, D. Esteve, and M.H. Devoret, Europhys. Lett. 17, 249 (1992).

${ }^{5}$ M. Switkes, C. Marcus, K. Capman, and A.C. Gossard, Science 283, 1905 (1999).

${ }^{6}$ B. Spivak, F. Zhou, and M.T. Beal Monod, Phys. Rev. B 51, 13226 (1995).

${ }^{7}$ P.W. Brouwer, Phys. Rev. B 58, R10 135 (1998).

${ }^{8}$ Q. Niu, Phys. Rev. Lett. 64, 1812 (1990).

${ }^{9}$ T.C.L.G. Sollner, et al., Appl. Phys. Lett. 43, 588 (1983).

${ }^{10}$ M. Büttiker, H. Thomas, and A. Prêtre, Z. Phys. B: Condens. Matter 94, 133 (1994).
${ }^{11}$ Our analysis can also be applied to a quantum dot if strong electron-electron interaction can be neglected. For example, one can consider a 2D quantum dot similar to the experiment of Ref. 5 , but with leads connected to the dot through narrow constrictions controlled by a gate voltage. Since the threshold of electron propagation in the constriction may be lower than that in the lead, the constrictions act like a double barrier whose height can be tuned by the gate voltage. However, to analyze such structure, one needs to perform two-dimensional calculations.

${ }^{12}$ M.K. Yip, J. Wang, and H. Guo, Z. Phys. B: Condens. Matter 104, 463 (1997).

${ }^{13}$ D.S. Fisher and P.A. Lee, Phys. Rev. B 23, 6851 (1981).

${ }^{14}$ If the double-barrier system is asymmetric, the transmission coefficient and hence pumping current will be suppressed.

${ }^{15}$ M. Buttiker, Phys. Rev. B 23, 1846 (1985); 33, 3020 (1986).

${ }^{16}$ Y.J. Wang, J. Wang, and H. Guo, Phys. Rev. B 47, 4348 (1993). 